\title{
A FAST DIRECT SOLVER FOR HIGH FREQUENCY SCATTERING FROM A LARGE CAVITY IN TWO DIMENSIONS
}

\author{
JUN LAI*, SIVARAM AMBIKASARAN*, AND LESLIE F. GREENGARD*†
}

\begin{abstract}
We present a fast direct solver for the simulation of electromagnetic scattering from an arbitrarily-shaped, large, empty cavity embedded in an infinite perfectly conducting half space. The governing Maxwell equations are reformulated as a well-conditioned second kind integral equation and the resulting linear system is solved in nearly linear time using a hierarchical matrix factorization technique. We illustrate the performance of the scheme with several numerical examples for complex cavity shapes over a wide range of frequencies.
\end{abstract}

Key words. electromagnetic scattering, fast direct solver, large cavity

1. Introduction. Electromagnetic scattering from large cavities has been studied extensively over the years [6, 7, 8, 14, 16, 11, 44, 50, due to the widespread presence of cavities in practical settings. It is of particular interest in radar cross section (RCS) analysis, both for accentuating a signal (in tracking a vehicle) and for its mitigation (in electromagnetic interference and stealth design). Cavities play an important role in these contexts because of the well-known fact that the intensity of the echo wave is often dominated by scattering from cavity-like components [47, such as the exhaust nozzle or engine inlet of an aircraft. In the context of design, we refer the reader to [12, 13, where RCS enhancement or reduction was carried out through the use of an optimization procedure based on a Newton-type method. At each iteration, a large-scale scattering problem has to be solved, dominating the net cost. A second application is nondestructive testing to determine the shape of an existing cavity. The corresponding stability analysis was initially studied in [15. Numerical inversion again requires an efficient solver that works over a range of frequencies. To obtain fine features inside the cavity, high frequency measurements are required, making the problems large, oscillatory and progressively more ill-conditioned. In short, efficient and accurate numerical methods for modeling in the presence of complex, arbitrarily-shaped cavities are becoming essential.

Integral equation methods are very natural choices for the solution of scattering problems because they discretize the scatterer alone and are able to impose outgoing radiation conditions without the need for truncating the spatial domain and imposing artificial boundary conditions. In the case of cavities in a conducting half-space, a variety of integral formulations exists and we refer the readers to Bao et al [6], Asvestas et al [10, Willers et al [58, Chandler-wilde et al [24] and the references therein for a complete discussion. Here, we simply note that the choice of integral equation has a great impact on the accuracy of the numerical discretization [41] and the condition number of the resulting linear system. In this article, we propose a boundary integral formulation that leads in a straightforward way to a well-posed, high-order discretization. Like the formulation of [6], we impose continuity conditions on a "transparent" dome covering the cavity, which reduces the problem to one posed in a bounded domain. A principal difference is that, in our case, we introduce a non-physical charge density on part of the ground plane which permits high order accuracy - avoiding the difficulties introduced by signularities at triple-points, points that lie at the intersection of more than two subdomains (see Fig. 1.1).

Historically, the major challenge with integral formulations has been that the corresponding linear system is dense so that solving by conventional linear solvers is expensive, requiring $\mathcal{O}\left(N^{3}\right)$ work for a $N \times N$ linear system. That complexity barrier was overcome by using iterative techniques based on Krylov subspace methods [9, 42, 56, 54, 57, 33, 32, coupled with fast matrix-vector product techniques such as the fast multipole method (FMM) 28, 38, 37, 27, tree based algorithms [17, panel clustering [40, FFT [31, wavelet based methods [51, and a host of others. Though these techniques have a number of attractions, the number of iterations required to achieve a specified accuracy is highly problem-dependent. In the context of electromagnetic scattering, if the geometry is complicated and if the frequency of the incident field is high, the number of iterations can be extremely large, so that the methods no longer behave linearly and require large amounts of storage. Recently, there has been an increasing focus on fast direct solvers [35] for dense linear systems arising from integral equations. This is an important and active area of research and we refer the reader to three relevant (and related) formualtions - those based on hierarchical off-diagonal

\footnotetext{
*Courant Institute of Mathematical Sciences, New York University, NY 10012

†Simons Center for Data Analysis, Simons Foundation, New York, NY 10010
} 
low-rank matrices (HODLR) 4, 5, 48, hierarchically semi-separable (HSS) or hierarchically block-separable (HBS) matrices [25, 26, 43, 52, 53], $\mathcal{H}$, and $\mathcal{H}^{2}$ matrices [18, 19, 20, 39]. In this article, since the integral equation we are solving is on a 1D manifold, we rely on the fast direct solver discussed in Ambikasaran and Darve [4, which scales almost linearly $\left(\operatorname{as} \mathcal{O}\left(N \log ^{2} N\right)\right)$ in the number of unknowns even for problems that are hundreds of wavelengths in size.

We restrict our attention here to time-harmonic scattering over a wide range of frequencies for a $2 \mathrm{D}$ cavity embedded in a ground plane, as shown in Figure 1.1. where the boundary of the cavity and the ground plane are perfectly conducting. The cavity is empty, i.e., the permittivity $\epsilon$ and permeability $\mu$ are constant everywhere inside the cavity and equal to that of the upper half-space.

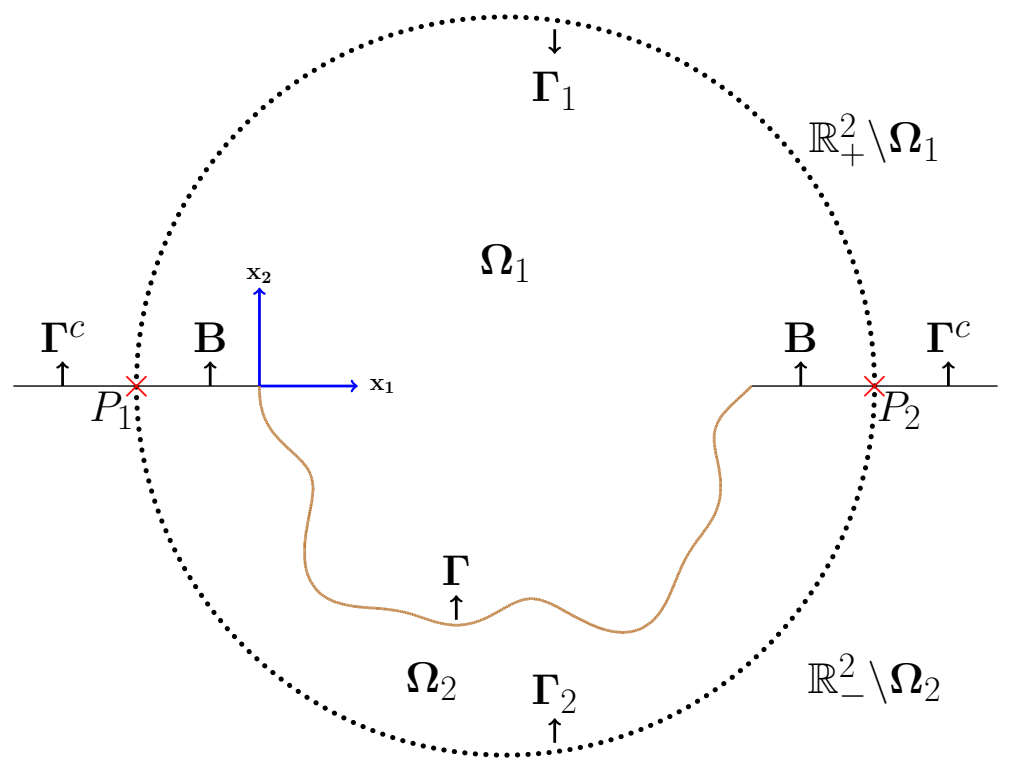

Fig. 1.1: Geometry of the cavity shape. Note that $P_{1}$ and $P_{2}$ are triple-points. They lie at the intersection of domains $\Omega_{1}, \Omega_{2}, R_{+}^{2} \backslash \Omega_{1}$, and $R_{-}^{2} \backslash \Omega_{2}$.

It is well known that, in 2D, the wave can be decomposed into a transverse magnetic (TM) polarization component and a transverse electric (TE) polarization component. Maxwell's equations reduce in this setting to a scalar Helmholtz equation for the $z$-component the magnetic (TE) or electric (TM) field, respectively. We restrict our attention to the TM polarization and denote the $z$-component of the electric field by $u$, but note that the treatment of the TE polarization is very similar.

We seek to determine the unknown scattered wave $u^{s}$, emanating from the cavity $\Omega_{1}$ in response to a known incident wave $u^{i}$. The governing equation is the Helmholtz equation:

$$
\left\{\begin{aligned}
\Delta u+k^{2} u & =0 \text { in } \Omega_{1} \cup R_{+}^{2} \\
u & =0 \text { on } \Gamma \cup \Gamma^{c} .
\end{aligned}\right.
$$

where $R_{+}^{2}$ denotes the upper half space, $\Gamma$ is the boundary of the cavity, $\Gamma^{c}$ is the ground plane and $k$ is the wavenumber. The wavenumber $k$ depends on the given angular frequency $\omega$ (time harmonic dependence is $e^{-i \omega t}$ ), i.e., $k=\omega \sqrt{\varepsilon \mu}$. where, as noted above, the permittivity $\epsilon$ and permeability $\mu$ are assumed to be constant.

The total field $u$ is considered as the summation of three parts [45]: the incident field $u^{i}$, the reflected field $u^{r}$ and the scattered field $u^{s} . u^{i}$ is known, and typically defined as a plane wave or the wave induced by known sources in the upper half-space. $u^{r}$ denotes the wave reflected by a perfectly conducting half-space (without a cavity) and can be computed analytically from $u^{i}$. The only unknown, therefore, is the scattered field $u^{s}$, which satisfies the Helmholtz equation (1.1) as well as the Sommerfeld radiation condition:

$$
\lim _{r \rightarrow \infty} \sqrt{r}\left(\frac{\partial u^{s}}{\partial r}-i k u^{s}\right)=0
$$


where $r=\sqrt{x_{1}^{2}+x_{2}^{2}}$.

The paper is organized as follows. In Section 2, we describe the cavity problem in more detail. We propose a new second kind integral formulation that leads to a stable numerical algorithm. In addition, we prove uniqueness for the equation, showing that the the formulation is well-posed. Section 3 discusses the numerical discretization of the integral equation, while Section 4 discusses the hierarchical, fast, direct solver that relies on specific properties of the matrix arising from discretization of our integral equation. Numerical examples are presented in Section 5, demonstrating the efficiency of the method and Section 6 contains some concluding remarks.

2. The cavity problem. Consider a time-harmonic wave incident on the cavity $\Omega_{1}$, embedded in an infinite ground plane $\Gamma^{c}$, illustrated in Fig. 1.1. The boundary components $\Gamma$ and $\Gamma^{c}$ are both assumed to be perfect conductors. We also assume that $\Gamma$ is defined by a piecewise smooth curve and that none of the corners between adjacent smooth components involve cusps (that is, the angle at each corner is greater than 0 and less than $2 \pi$ ).

When the incoming field is a plane wave, we have $u^{i}=e^{i k\left(\cos \theta x_{1}-\sin \theta x_{2}\right)}$, and $u^{r}=e^{i k\left(\cos \theta x_{1}+\sin \theta x_{2}\right)}$, where $\theta$ is the angle of incidence with respect to the positive $x_{1}$ axis. In this case, the scattered wave $u^{s}$ satisfies the equation:

$$
\left\{\begin{aligned}
\Delta u^{s}+k^{2} u^{s} & =0 \text { in } \Omega_{1} \cup R_{+}^{2} \\
u^{s} & =g \text { on } \Gamma \cup \Gamma^{c}
\end{aligned}\right.
$$

along with the Sommerfeld radiation condition (1.2), where $g=-\left(u^{i}+u^{r}\right)$.

2.1. The integral formulation. A variety of integral formulations have been proposed in the literature for the cavity problem. Those discussed in [6, 58, for example, are based on Green's identities. Another formulation, based on the method of images, is discussed in [10. Our formulation is based on potential theory, with the goal of deriving a second kind integral equation that will lead to a high order numerical scheme, even in the presence of sharp corners. In all these approaches, it is convenient to introduce an artificial interface close to the cavity, which we denote here by $\Gamma_{1}$ (illustrated by the dotted line in Fig. 1.1. One does not need to introduce artificial boundary conditions on this interface, however. One simply imposes continuity conditions so that there is a representation for the scattered wave in the exterior of $\Gamma_{1}$ as an integral over the artificial interface itself. $\Gamma_{1}$ needs to be chosen so that its reflection with respect to the ground plane, $\Gamma_{2}$, does not intersect the cavity. We denote by $B$ the part of the ground plane $\Gamma^{c}$ that connects $\Gamma_{1}$ and $\Gamma$ and define $\Omega_{1}$ to be the domain enclosed by $\Gamma \cup B \cup \Gamma_{1}$. Its complement is the unbounded domain $R_{+}^{2} \backslash \Omega_{1}$.

We let $\Phi(\mathbf{x}, \mathbf{y})$ denote the (radiating) free space Green's function for the Helmholtz equation and we let $\Phi^{H}(\mathbf{x}, \mathbf{y})$ denote the Green's function satisfying homogeneous Dirichlet conditions on the half space. It is well-known that

$$
\begin{aligned}
\Phi(\mathbf{x}, \mathbf{y}) & =\frac{i}{4} H_{0}^{(1)}(k|\mathbf{x}-\mathbf{y}|) \\
\Phi^{H}(\mathbf{x}, \mathbf{y}) & =\frac{i}{4} H_{0}^{(1)}(k|\mathbf{x}-\mathbf{y}|)-\frac{i}{4} H_{0}^{(1)}\left(k\left|\mathbf{x}-\mathbf{y}^{\prime}\right|\right),
\end{aligned}
$$

where $H_{0}^{(1)}$ is the Hankel function of the first kind of order zero and $\mathbf{y}^{\prime}=\left(y_{1},-y_{2}\right)$ is the image point for $\mathbf{y}=\left(y_{1}, y_{2}\right)$.

A natural representation for $u^{s}$ in the unbounded domain $R_{+}^{2} \backslash \Omega_{1}$ is

$$
u^{s}=\mathcal{S}_{\Gamma_{1}}^{H} \sigma+\mathcal{D}_{\Gamma_{1}}^{H} \mu, \text { for } \mathbf{x} \in R_{+}^{2} \backslash \Omega_{1}
$$

where $\mathcal{S}^{H}$ and $\mathcal{D}^{H}$ are single and double layer potentials, using the half-space Green's function:

$$
\begin{aligned}
\mathcal{S}_{\Gamma_{1}}^{H} \sigma & =\int_{\Gamma_{1}} \Phi^{H}(\mathbf{x}, \mathbf{y}) \sigma(\mathbf{y}) d s_{\mathbf{y}} \\
\mathcal{D}_{\Gamma_{1}}^{H} \mu & =\int_{\Gamma_{1}} \frac{\partial \Phi^{H}(\mathbf{x}, \mathbf{y})}{\partial n(\mathbf{y})} \mu(\mathbf{y}) d s_{\mathbf{y}} .
\end{aligned}
$$


Here the unit normal vector $n$ on $\Gamma_{1}$ is assumed to be oriented toward the exterior of $\Omega_{1} . \sigma$ and $\mu$ are, for the moment, unknown density functions on the boundary $\Gamma_{1}$.

REMARK 1. By the methods of images, we can rewrite $\mathcal{S}^{H}$ as:

$$
\mathcal{S}_{\Gamma_{1}}^{H} \sigma=\mathcal{S}_{\Gamma_{1}} \sigma-\mathcal{S}_{\Gamma_{2}} \sigma
$$

where $S_{\Gamma}$ denotes single layer potential based on the free-space Green's function

$$
S_{\Gamma} \sigma=\int_{\Gamma} \Phi(\mathbf{x}, \mathbf{y}) \sigma(\mathbf{y}) d s_{\mathbf{y}},
$$

and for $\mathbf{x}^{\prime} \in \Gamma_{2}, \sigma\left(\mathbf{x}^{\prime}\right)=\sigma(\mathbf{x})$, where $\mathbf{x}^{\prime}$ is the image point for $\mathbf{x}$. Clearly, a similar expression holds for $\mathcal{D}^{H}$ using the free-space double layer potential

$$
D_{\Gamma} \mu=\int_{\Gamma} \frac{\partial \Phi(\mathbf{x}, \mathbf{y})}{\partial n(\mathbf{y})} \mu(\mathbf{y}) d s_{\mathbf{y}} .
$$

Suppose now that we represent the scattered field for $\mathbf{x} \in \Omega_{1}$ by

$$
u^{s}(\mathbf{x})=\mathcal{S}_{\Gamma_{1}} \sigma+\mathcal{D}_{\Gamma_{1}} \mu+\mathcal{D}_{B} \mu+\mathcal{D}_{\Gamma} \mu .
$$

Imposing the continuity of the potential $u$ and its normal derivative across $\Gamma_{1}$, and using the representations (2.4) and (2.8), leads to

$$
\left\{\begin{array}{c}
\mu-\mathcal{S}_{\Gamma_{2}} \sigma-\mathcal{D}_{\Gamma_{2}} \mu-\mathcal{D}_{B} \mu-\mathcal{D}_{\Gamma} \mu=0, \quad \text { for } \mathbf{x} \in \Gamma_{1} \\
\sigma+\mathcal{N}_{\Gamma_{2}} \sigma+\mathcal{T}_{\Gamma_{2}} \mu+\mathcal{T}_{B} \mu+\mathcal{T}_{\Gamma} \mu=0,
\end{array}\right.
$$

For this, one needs to use standard jump relations for the layer potentials $\mathcal{S}$ and $\mathcal{D}$ [29, 30]. The operators $\mathcal{N}$ and $\mathcal{T}$ are defined as the normal derivatives of $\mathcal{S}$ and $\mathcal{D}$, respectively:

$$
\begin{aligned}
\mathcal{N}_{\Gamma} \sigma & =\int_{\Gamma} \frac{\partial \Phi(\mathbf{x}, \mathbf{y})}{\partial n(\mathbf{x})} \sigma(\mathbf{y}) d s_{\mathbf{y}} \\
\mathcal{T}_{\Gamma} \mu & =\int_{\Gamma} \frac{\partial^{2} \Phi(\mathbf{x}, \mathbf{y})}{\partial n(\mathbf{x}) \partial n(\mathbf{y})} \mu(\mathbf{y}) d s_{\mathbf{y}} .
\end{aligned}
$$

Note that the operator $\mathcal{T}$ is hypersingular on $\Gamma$, with its value interpreted in the Hadamard finite part sense. Similarly, using the representation (2.8), and letting $x$ approach the boundary $\Gamma \cup B$, yields

$$
-\frac{1}{2} \mu+\mathcal{S}_{\Gamma_{1}} \sigma+\mathcal{D}_{\Gamma_{1}} \mu+\mathcal{D}_{B} \mu+\mathcal{D}_{\Gamma} \mu=g, \text { for } \mathbf{x} \in \Gamma \cup B .
$$

Combining eqs. 2.9) and 2.12 we obtain a closed system for $\sigma$ and $\mu$. Unfortunately, careful analysis shows that it is not a Fredholm equation of the second kind. In particular, the hypersingular operators are unbounded at the triple-points $P_{1}$ and $P_{2}$ in Fig. 1.1. This leads to a failure of convergence. Following the approach of [34, 36, we remedy the situation by using a non-physical representation near triple-points so that the resulting integral equation involves only the difference of two hypersingular kernels, which is easily seen to be compact. Thus, we propose the following, new formulation:

$$
\left\{\begin{array}{lll}
u^{s}=\mathcal{S}_{\Gamma_{1}}^{H} \sigma+\mathcal{D}_{\Gamma_{1}}^{H} \mu+\mathcal{D}_{B} \mu & & \text { for } \mathbf{x} \in R_{+}^{2} \backslash \Omega_{1} \\
u^{s}=\mathcal{S}_{\Gamma_{1}}^{H} \sigma+\mathcal{D}_{\Gamma_{1}}^{H} \mu+\mathcal{D}_{B} \mu+\mathcal{D}_{\Gamma} \mu & & \text { for } \mathbf{x} \in \Omega_{1}
\end{array}\right.
$$

We refer to the representation as non-physical because the terms we have added $\left(\mathcal{D}_{B} \mu\right.$ for the exterior field and $-\mathcal{S}_{\Gamma_{2}} \sigma-\mathcal{D}_{\Gamma_{2}} \mu$ for the interior field) that involve boundary components that do not actually impinge on the domain. The advantage we obtain is a cancellation of the hypersingular terms. To see this, imposing the continuity and boundary conditions as above, and using the standard jump relations, we obtain the system:

$$
\left\{\begin{array}{rlrl}
\mu-\mathcal{D}_{\Gamma} \mu & =0, & & \text { for } \mathbf{x} \in \Gamma_{1} \\
\sigma+\mathcal{T}_{\Gamma} \mu & =0, & & \text { for } \mathbf{x} \in \Gamma_{1} \\
-\frac{1}{2} \mu+\mathcal{S}_{\Gamma_{1}}^{H} \sigma+\mathcal{D}_{\Gamma_{1}}^{H} \mu+\mathcal{D}_{B} \mu+\mathcal{D}_{\Gamma} \mu & =g, & & \text { for } \mathbf{x} \in B \cup \Gamma
\end{array} .\right.
$$


Although the representation $(2.13)$ is slightly more involved than (2.4) and (2.8), note that the resulting system of integral equations is actually simpler. In particular, the first two equations in (2.14) imply that $\mu$ and $\sigma$ on $\Gamma_{1}$ are directly determined by the value of $\mu$ on $\Gamma$. More importantly, the system is a Fredholm equation of the second kind in a suitably-defined space. The analysis is somewhat technical, since operators that are compact on smooth domains are only bounded on domains with corners. That is the case here, and we refer the reader to [21] for further details. In the next section, we discuss existence and uniqueness, followed by a discussion of numerical discretization.

REMARK 2. In [10], a related formulation was developed that used only the unknown $\mu$ on $B \cup \Gamma$ in 2.13). This, however, requires that the cavity remain strictly below the ground plane. Our approach works for an arbitrary cavity shape.

2.2. Existence and uniqueness. To simplify the analysis, we assume that the boundary of the cavity $B \cup \Gamma, \Gamma_{1}$ and $\Gamma_{2}$ are $C^{2}$ smooth curves, in which case the solution $u$ to eq. 2.1 lies in $C^{2}\left(R_{+}^{2} \cup \Omega_{1}\right) \cup$ $C\left(\overline{R_{+}^{2} \cup \Omega_{1}}\right)$ (see [58]) so that the standard Fredholm theory applies. Thus, to establish uniqueness for the system (2.14), it suffices to show that $\mu=0$ and $\sigma=0$ if $g=0$. Extension of the proof to the piecewisesmooth case is straightforward, and consists largely in a switch to the corresponding Sobolev space in order that the Fredholm theory be applicable.

THEOREM 2.1. Given $k>0$, the system

$$
\left\{\begin{aligned}
\mu-\mathcal{D}_{\Gamma} \mu & =0, & & \text { for } \mathbf{x} \in \Gamma_{1} \\
\sigma+\mathcal{T}_{\Gamma} \mu & =0, & & \text { for } \mathbf{x} \in \Gamma_{1} \\
-\frac{1}{2} \mu+\mathcal{S}_{\Gamma_{1}}^{H} \sigma+\mathcal{D}_{\Gamma_{1}}^{H} \mu+\mathcal{D}_{B} \mu+\mathcal{D}_{\Gamma} \mu & =0, & & \text { for } \mathbf{x} \in B \cup \Gamma
\end{aligned}\right.
$$

has only the trivial solution for $\mu \in C(B) \cup C(\Gamma) \cup C\left(\Gamma_{1}\right)$ and $\sigma \in C\left(\Gamma_{1}\right)$.

Proof. Motivated by the analysis of [6], we let

$$
v=\left\{\begin{array}{l}
\mathcal{S}_{\Gamma_{1}}^{H} \sigma+\mathcal{D}_{\Gamma_{1}}^{H} \mu+\mathcal{D}_{B} \mu, \text { for } \mathbf{x} \in R^{2} \backslash\left(\Omega_{1} \cup \Omega_{2}\right) \\
\mathcal{S}_{\Gamma_{1}}^{H} \sigma+\mathcal{D}_{\Gamma_{1}}^{H} \mu+\mathcal{D}_{B} \mu+\mathcal{D}_{\Gamma} \mu, \text { for } \mathbf{x} \in \Omega_{1} \cup \Omega_{2}
\end{array}\right.
$$

where $\Omega_{2}$ is the area enclosed by $\Gamma \cup B \cup \Gamma_{2}$.

Combining 2.16) and eq. 2.15, we have

$$
v=0 \text { on } \Gamma^{c}, \quad \lim _{\substack{x \in \Omega_{1} \\ x \rightarrow \Gamma \cup B}} v(x)=0,
$$

and the jump conditions

$$
\begin{aligned}
{\left.[v]\right|_{\Gamma_{1}} } & =0 \\
{\left.\left[\frac{\partial v}{\partial n}\right]\right|_{\Gamma_{1}} } & =0
\end{aligned}
$$

where $\left.[v]\right|_{\Gamma_{1}}$ denotes the jump of the function $v$ across the boundary $\Gamma_{1}$.

We first prove $v$ is identically zero in $R_{+}^{2} \cup \Omega_{1}$. For this, choose a sufficiently large half disk $D$ above the ground plane that contains the boundary $\Gamma_{1}$. For the area $D \backslash \Omega_{1}$, the boundary consists of $\Gamma_{1}$, part of the ground plane (still denoted by $\Gamma^{c}$ ) and a half circle, denoted by $\partial D$. Applying Green's theorem to $v$ in $D \backslash \Omega_{1}$ and $\Omega_{1}$, respectively, we obtain

$$
\int_{\Omega_{1}} v \triangle \bar{v}+\nabla v \nabla \bar{v} d x=\int_{B \cup \Gamma} v \frac{\partial \bar{v}}{\partial n} d s+\int_{\Gamma_{1}} v \frac{\partial \bar{v}}{\partial n} d s
$$

and

$$
\int_{D \backslash \Omega_{1}} v \triangle \bar{v}+\nabla v \nabla \bar{v} d x=\int_{\partial D} v \frac{\partial \bar{v}}{\partial n} d s-\int_{\Gamma_{1}} v \frac{\partial \bar{v}}{\partial n} d s+\int_{\Gamma^{c}} v \frac{\partial \bar{v}}{\partial n} d s .
$$

Adding eq. 2.19) and eq. 2.20, together with 2.17) and 2.18, yields

$$
\int_{\partial D} v \frac{\partial \bar{v}}{\partial n} d s=\int_{D \backslash \Omega_{1}}\left(-k^{2} v \bar{v}+\nabla v \nabla \bar{v}\right) d x+\int_{\Omega_{1}}\left(-k^{2} v \bar{v}+\nabla v \nabla \bar{v}\right) d x
$$


which implies that

$$
\operatorname{Im}\left(\int_{\partial D} v \frac{\partial \bar{v}}{\partial n} d s\right)=0
$$

It follows from Rellich's theorem [29, applied to the half space, and the unique continuation property [30], that $v=0$ in $R_{+}^{2} \cup \Omega_{1}$.

We next show $v(x)$ satisfies the following equation in $\Omega_{2}$

$$
\left\{\begin{aligned}
\Delta v+k^{2} v & =0, \text { for } x \in \Omega_{2} \\
v & =\mathcal{D}_{\Gamma} \mu+\mathcal{D}_{\Gamma^{\prime}} \mu, \quad \text { on } \Gamma_{2} \\
\frac{\partial v}{\partial n} & =\mathcal{T}_{\Gamma} \mu+\mathcal{T}_{\Gamma^{\prime}} \mu, \quad \text { on } \Gamma_{2} \\
v & =\mu, \quad \text { on } B \cup \Gamma \\
\frac{\partial v}{\partial n} & =0, \quad \text { on } B \cup \Gamma,
\end{aligned}\right.
$$

where $\Gamma^{\prime}$ is the image curve of $\Gamma$ with respect to the ground plane and $\mu(\mathbf{x})$ on $\Gamma^{\prime}$ is the image source of $\mu\left(\mathbf{x}^{\prime}\right)$ on $\Gamma$.

It follows from (2.16) that we have the following jump condition on $\Gamma_{2}$ :

$$
\begin{array}{r}
{[v]_{\Gamma_{2}}=\mu+\mathcal{D}_{\Gamma} \mu} \\
{\left[\frac{\partial v}{\partial n}\right]_{\Gamma_{2}}=-\sigma+\mathcal{T}_{\Gamma} \mu .}
\end{array}
$$

Since $v=0$ in $R_{+}^{2} \cup \Omega_{1}$, by symmetry, $v=0$ in $R^{2} \backslash\left(\Omega_{1} \cup \Omega_{2}\right)$. From 2.15$), \mu(\mathbf{x})=\mu\left(\mathbf{x}^{\prime}\right)=\mathcal{D}_{\Gamma} \mu\left(\mathbf{x}^{\prime}\right)$ for $\mathbf{x} \in \Gamma_{2}$. However, by the definition of the image curve $\Gamma^{\prime}$ and the source $\mu$ on $\Gamma^{\prime}$, it is easy to see that $\mathcal{D}_{\Gamma} \mu\left(\mathbf{x}^{\prime}\right)=\mathcal{D}_{\Gamma^{\prime}} \mu(\mathbf{x})$. Similarly, one can see that $\sigma(\mathbf{x})=-\mathcal{T}_{\Gamma^{\prime}} \mu(\mathbf{x})$ for $\mathbf{x} \in \Gamma_{2}$. Combining eqs 2.15] and 2.24 leads to

$$
\begin{aligned}
& \lim _{\substack{\mathbf{x} \in \Omega_{2} \\
\mathbf{x} \rightarrow \Gamma_{2}}} v=\mathcal{D}_{\Gamma} \mu+\mathcal{D}_{\Gamma^{\prime}} \mu, \\
& \lim _{\substack{\mathbf{x} \in \Omega_{2} \\
\mathbf{x} \rightarrow \Gamma_{2}}} \frac{\partial v}{\partial n}=\mathcal{T}_{\Gamma} \mu+\mathcal{T}_{\Gamma^{\prime}} \mu .
\end{aligned}
$$

We therefore obtain the first two boundary conditions for $v$ in 2.23 . The boundary conditions on $B \cup \Gamma$ are obtained from the jump condition

$$
\begin{aligned}
{[v]_{B \cup \Gamma} } & =\mu \\
{\left[\frac{\partial v}{\partial n}\right]_{B \cup \Gamma} } & =0,
\end{aligned}
$$

and the fact that $v$ is zero in $\Omega_{1}$.

We now prove that the solution to 2.23 is identically zero. Let

$$
w(x)=\mathcal{D}_{\Gamma} \mu+\mathcal{D}_{\Gamma^{\prime}} \mu, \quad \forall x \in R_{-}^{2} \backslash \Omega_{2} .
$$

It is easy to see $\frac{\partial w(x)}{\partial n}=0$ on $\Gamma^{c}$ and

$$
\begin{aligned}
w\left(x^{+}\right)-v\left(x^{-}\right) & =0, \\
\frac{\partial w\left(x^{+}\right)}{\partial n}-\frac{\partial v\left(x^{-}\right)}{\partial n} & =0
\end{aligned}
$$

where $w\left(x^{+}\right)$and $v\left(x^{-}\right)$denote the limiting function values as $x$ approaches $\Gamma_{2}$ from $R_{-}^{2} \backslash \Omega_{2}$ and $\Omega_{2}$, respectively.

As in the first part of the proof, we now choose a sufficiently large half disk, still denoted by $D$, in $R_{-}^{2} \backslash \Omega_{2}$ that contains $\Gamma_{2}$. Applying the first Green's theorem to $D \backslash \Omega_{2}$ and $\Omega_{2}$, eq. $(2.23)$, and the various jump conditions above lead to:

$$
\operatorname{Im}\left(\int_{\partial \Omega_{2}} w \frac{\partial \bar{w}}{\partial n}\right)=0
$$


Once again, from Rellich's theorem and unique continuation we may conclude that $v=0$ in $\Omega_{2}$, which implies that (a) $\mu=0$ on $B \cup \Gamma$ from 2.23), and (b) $\mu=0, \sigma=0$ on $\Gamma_{1}$ from 2.15). This completes the proof.

REMARK 3. The cavity problem can also be solved through a variational formulation. The corresponding well-posedness was studied in [7, 8].

3. Numerical discretization. For our numerical simulations, we will make use of Nyström discretization of the integral equation 2.14. Since the kernels in the integral equation are logarithmically singular, this requires some care. Fortunately, for smooth boundaries, there are by now a host of simple, high-order rules available (see, for example, 1, 22, 41, 46]). Here, we use composite Gaussian quadrature. Following the discussion of [34, we divide each smooth component of the boundary into $N$ curved panels with $p$ points in each panel. The $p$ points are chosen as scaled Gaussian-Legendre nodes, so that for smooth integrands the order of accuracy is $(2 p-1)$. More precisely, we replace the integral

$$
\int_{\Gamma} K(\mathbf{x}, \mathbf{y}) \sigma(\mathbf{y}) d S_{\mathbf{y}}
$$

by the quadrature

$$
\sum_{i=1}^{N} \sum_{j=1}^{p} \mathcal{K}\left(\mathbf{x}_{l, m}, \mathbf{y}_{i, j}\right) \sigma\left(\mathbf{y}_{i, j}\right) w_{l, m, i, j}
$$

where $\mathbf{x}_{l, m}$ is the $m$-th Gauss-Legendre node on panel $l, \mathbf{y}_{i, j}$ is the $j$-th Gauss-Legendre node on panel $i$, $w_{l, m, i, j}$ is the quadrature weight and $\mathcal{K}$ is the "quadrature kernel". For non-adjacent panels, we simply set $\mathcal{K}\left(\mathbf{x}_{l, m}, \mathbf{y}_{i, j}\right)=K\left(\mathbf{x}_{l, m}, \mathbf{y}_{i, j}\right)$, for node $\mathbf{x}_{l, m}$ on panel $l$ and node $\mathbf{y}_{i, j}$ on panel $j$. The weights $w_{l, m, i, j}$ in that case are the standard Gauss-Legendre weights at $\mathbf{y}_{i, j}$ scaled to the length of the $i$-th panel. For the selfinteraction of a panel $(i=l)$, or the interaction with an adjacent panel, the quadrature kernel is computed by generalized Gaussian quadrature [22. From a linear algebra point of view, the self and adjacent panel interactions correspond to the block tridiagonal entries in the matrix. In our direct solver, these elements are precomputed and stored. All other matrix entries are computed on the fly.

In the presence of corners, a graded mesh [21, 41] is used to maintain high order accuracy. More specifically, after uniform discretization by panels, we perform a dyadic refinement for the panels that impinges on each corner point. A $p$-th order generalized Gaussian quadrature is used on each of the refined panels. The formal error analysis for such discretization is rather involved, since it depends on the regularity of $\sigma$ and $\mu$. Readers are referred to [21, 23, 41] and the references therein for a discussion of the relevant analysis. Typically, if $\epsilon$ denotes the length of the finest panel in the refinement, then the error is proportional to $\mathcal{O}\left(e^{-p}+\epsilon\right)$. Since the mesh is dyadically refined, this requires $\mathcal{O}(\log (1 / \epsilon))$ additional nodes at each corner.

With a total of $N$ nodes, solving the linear system corresponding to the above discretization by conventional Gaussian elimination requires $\mathcal{O}\left(N^{3}\right)$ work - an arduous task if the cavity has a complicated boundary or if the wavenumber of the incoming field is high. If the wave number $k=1000$, for example, and if the length of the boundary is 20 (in normalized units), then the number of unknowns is around 100,000, assuming 20 points per wavelength and counting each complex unknown as two real unknowns. Conventional solvers would take a few days to factor this system on a single core machine operating at $2.6 \mathrm{GHz}$. The fast direct solver described in the next section takes around $10-15$ minutes.

4. Fast direct solver. In this section, we briefly outline the fast direct solver we will use to solve the discretized integral equation (2.14). It is a simple extension of the method described in [4, to which we refer the reader for a more complete description of the method. As noted in the introduction, there are two strong arguments in favor of this strategy:

- The performance of the direct solver is insensitive to multiple reflections inside the cavity. At high frequencies, this causes the problem to be physically ill-conditioned and causes severe degradation in the convergence of iterative methods.

- The solver is particularly effective for multiple right hand sides. It proceeds in two steps: first, the construction of a fast hierarchical factorization, and second the application of the factored inverse to each new right-hand side, at much lower cost. 
4.1. Hierarchical off-diagonal low-rank matrices. Discretizing the integral equation 2.9 along the curve (a one-dimensional manifold) and ordering the unknowns and equations sequentially, yields a linear system of the form $A \sigma=b$, where $A \in \mathbb{R}^{N \times N}, \sigma, b \in \mathbb{R}^{N}$. Because potential-theoretic interactions are smooth in the far field, the matrix $A$ has a hierarchical off-diagonal low-rank (HODLR) structure, as observed in [4, 5, 48. (Related formalisms that also permit fast solution can be found in [25, 26, 43, 52, 53, [18, 19, 20, 39, 2], and the references therein.)

For illustration, we note that a 2-level HODLR matrix can be written in the form shown in equation 4.2 .

$$
\begin{aligned}
A & =\left[\begin{array}{cc}
A_{1}^{(1)} & U_{1}^{(1)} K_{1,2}^{(1)} V_{2}^{(1)^{T}} \\
U_{2}^{(1)} K_{2,1}^{(1)} V_{1}^{(1)^{T}} & A_{2}^{(1)}
\end{array}\right] \\
& =\left[\begin{array}{ccc}
{\left[\begin{array}{cc}
A_{1}^{(2)} & U_{1}^{(2)} K_{1,2}^{(2)} V_{2}^{(2)^{T}} \\
U_{2}^{(2)} K_{2,1}^{(2)} V_{1}^{(2)^{T}} & A_{2}^{(2)}
\end{array}\right]} & U_{1}^{(1)} K_{1,2}^{(1)} V_{2}^{(1)^{T}} \\
U_{2}^{(1)} K_{2,1}^{(1)} V_{1}^{(1)^{T}} & {\left[\begin{array}{cc}
A_{3}^{(2)} & U_{3}^{(2)} K_{3,4}^{(2)} V_{4}^{(2)^{T}} \\
U_{4}^{(2)} K_{4,3}^{(2)} V_{3}^{(2)^{T}} & A_{4}^{(2)}
\end{array}\right]}
\end{array}\right]
\end{aligned}
$$

Each off-diagonal block is of low-rank (i.e., the rank of these blocks does not grow with the system size), although these ranks can all be different. In general, for a $\kappa$-level HODLR matrix $A$, the $i^{\text {th }}$ diagonal block at level $k$, where $1 \leq i \leq 2^{k}$ and $0 \leq k<\kappa$, denoted by $A_{i}^{(k)}$, can be written as

$$
A_{i}^{(k)}=\left[\begin{array}{cc}
A_{2 i-1}^{(k+1)} & U_{2 i-1}^{(k+1)} K_{2 i-1,2 i}^{(k+1)} V_{2 i}^{(k+1)^{T}} \\
U_{2 i}^{(k+1)} K_{2 i, 2 i-1}^{(k+1)} V_{2 i-1}^{(k+1)^{T}} & A_{2 i}^{(k+1)}
\end{array}\right],
$$

where $A_{i}^{(k)} \in \mathbb{R}^{N / 2^{k} \times N / 2^{k}}$, and $U_{2 i-1}^{(k)}, U_{2 i}^{(k)}, V_{2 i-1}^{(k)}, V_{2 i}^{(k)}$ are thin matrices with $\frac{N}{2^{k}}$ rows. A pictorial representation of the matrix $A$ is shown in Figure 4.1 .
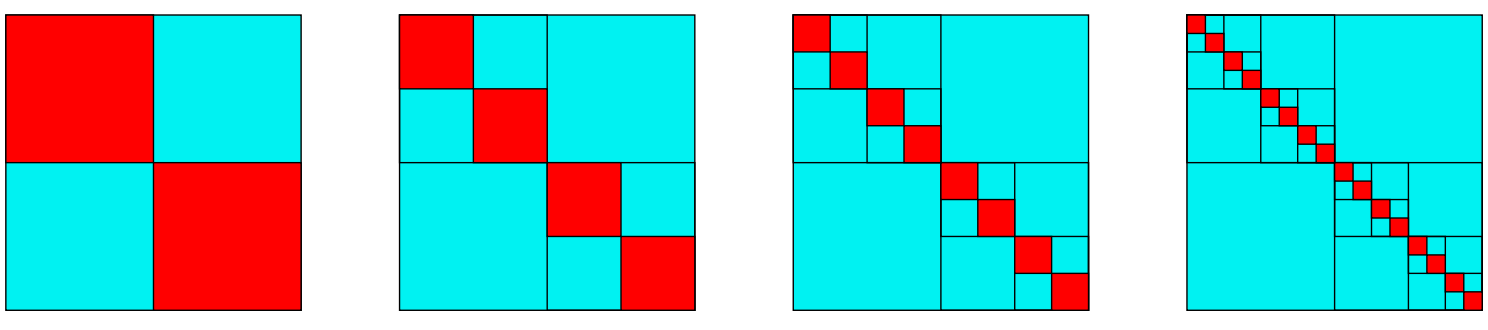

$\rightarrow$ Full rank;

$\square \rightarrow$ Low rank;

Fig. 4.1: A hierarchical off-diagonal low-rank matrix at different levels.

For the solver developed here, the low-rank decomposition of the off-diagonal blocks is obtained using the adaptive cross approximation (ACA) [55, 59] algorithm, which is a modification of the partially pivoted $\mathrm{LU}$ algorithm. The advantage of ACA is that the computational cost of obtaining a low-rank factorization of a low-rank $M \times N$ matrix is $\mathcal{O}_{\epsilon}(M+N)$. The technique is based entirely on numerical linear algebra, so the low-rank construction of the off-diagonal blocks is independent of the underlying integral operator. Once the low-rank decomposition of the off-diagonal blocks at all levels is obtained, the factorization of the matrix $A$ proceeds along the lines described in [4]. In other words, the matrix $A$ is factored as shown in eq. 4.4.

$$
A=A_{\kappa} A_{\kappa-1} \cdots A_{1} A_{0},
$$

where the $A_{i}$ 's are block diagonal matrices with $2^{i}$ diagonal blocks and each block is a low-rank perturbation of the identity matrix. The factorization can be obtained at a computational cost of the order $\mathcal{O}\left(N \log ^{2} N\right)$ by recursive application of the Sherman-Morrison-Woodbury formula. A pictorial representation of the 


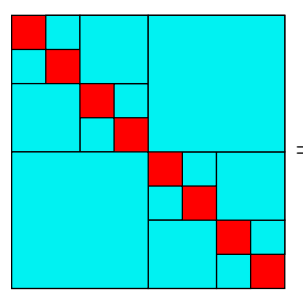

$A$

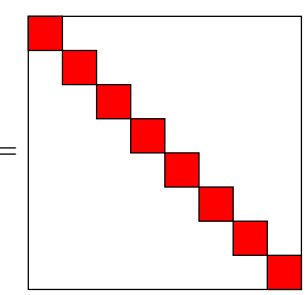

$A_{3}$

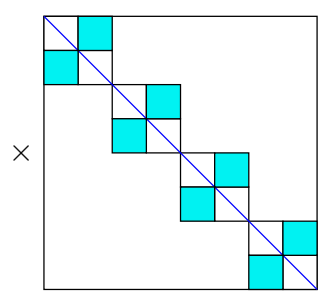

$A_{2}$

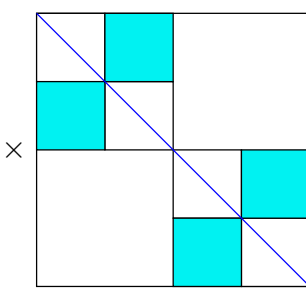

$A_{1}$

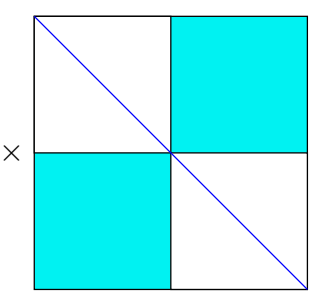

$A_{0}$

Full rank;

Low-rank;

Identity matrix;

Zero matrix;

Fig. 4.2: Factorization of a HODLR matrix at level 3.

above factorization for a level 3 HODLR matrix is shown in Figure 4.2. Other fast methods, such as the interpolative decomposition 49 can be used in place of ACA. Further details can be found in 4 and an implementation is available from 3 .

As with any direct factorization, a principal advantage is the performance of the scheme with multiple right hand sides. That is, the cost of solving the linear system with ' $r$ ' right-hand sides scales as $\mathcal{O}(r N \log N)$, with a smaller constant than for the factorization step. This is very attractive in the present context, since it permits the computation of the scattered field for multiple incident angles in a negligible amount of time. Each problem corresponds to a new right-hand side in the integral equation.

5. Numerical results. In this section, we illustrate the performance of our algorithm on three different cavity shapes. In general, there is no exact solution for the field scattering from a cavity embedded in a ground plane. We may, however, validate our solver by using "artificial" boundary data generated by a collection of point sources, in which case the potential field is known and can be used for comparison with the potential generated by the integral representation on the boundary. More specifically, we define the following field in the perturbed upper half-space $R_{+}^{2} \cup \Omega_{1}$ :

$$
u(\mathbf{x})=\frac{i}{4} H_{0}^{(1)}\left(k\left|\left(\mathbf{x}-\mathbf{x}_{\mathbf{0}}\right)\right|\right)+\frac{i}{4} H_{0}^{(1)}\left(k\left|\left(\mathbf{x}-\mathbf{x}_{\mathbf{0}}^{\prime}\right)\right|\right) .
$$

We then solve the following system of equations

$$
\left\{\begin{aligned}
-\mu+\mathcal{D}_{\Gamma} \mu & =u(\mathbf{x}), & & \text { for } \mathbf{x} \in \Gamma_{1} \\
\sigma+\mathcal{T}_{\Gamma} \mu & =\frac{\partial u(\mathbf{x})}{\partial n}, & & \text { for } \mathbf{x} \in \Gamma_{1} \\
-\frac{1}{2} \mu+\mathcal{S}_{\Gamma_{1}}^{H} \sigma+\mathcal{D}_{\Gamma_{1}}^{H} \mu+\mathcal{D}_{B} \mu+\mathcal{D}_{\Gamma} \mu & =u(\mathbf{x}), & & \text { for } \mathbf{x} \in B \cup \Gamma
\end{aligned}\right.
$$

where $\mathbf{x}_{\mathbf{0}}=(5,12), \mathbf{x}_{\mathbf{0}}^{\prime}=(5,-12)$ and the center of the cavity is at $(0.5,0)$. It is straightforward to see that the field given by the representation $(2.13)$ should be equal to $u(x)$ in $\Omega_{1}$ and zero in $R_{+}^{2} \backslash \Omega_{1}$.

When presenting the numerical results, we use the following notation:

- $N_{m i d}$ : Number of equal-sized curve segments used to discretize each smooth component of the piecewise-smooth boundary.

- $N_{\text {corner }}$ : Number of panels used to dyadically refine the first and last panel on each smooth component.

- $N_{t o t}$ : Total number of unknowns in the system

- $T_{\text {factor }}$ : Amount of time in second to factorize the system

- $T_{\text {solve }}$ : Amount of time in second required to solve the system after factorization

- $E_{\text {error }}$ : The average relative error at some random points in $\Omega_{1}$.

In the examples below, when solving the true scattering problem, the incoming field is assumed to be a plane wave and we compute the backscatter radar cross section (RCS) for each cavity. The backscatter RCS is defined as the intensity of the far field pattern in the same direction as the incident angle. When 
the boundary of the cavity is strictly below the ground plane, it can be shown the backscatter RCS is given by [45]:

$$
R C S=\frac{4}{k}\left|\frac{k}{2} \sin \theta \int_{\Gamma} e^{i k \cos \theta x} d x\right|^{2}
$$

where $\theta$ is the angle of incidence and $\Gamma$ is the aperture of the cavity. If part of the boundary is above the ground plane, the RCS can be found from (2.13) and the asymptotic behavior of the Hankel function.

We choose the artificial boundary to be a half circle centered at $(0.5,0)$, the center of the aperture, with a radius of 2.5. For numerical stability, we use inner-product-preserving Nyström scaling, as proposed in 21. That is, the discrete unknowns are taken to be the physical unknowns multiplied by the square root of the corresponding quadrature weight. All numerical tests have been carried out on a laptop with $4 \mathrm{~Gb}$ memory and $2.6 \mathrm{GHz}$ Intel CPU.

5.1. Example 1: Pot shaped cavity. In our first example, we consider the pot shaped cavity shown in Figure 5.1. The width of the aperture is 1 , below which is a circle of radius $\sqrt{2} / 2$ centered at $(0.5,-1)$. The accuracy of the solution for wavenumbers ranging from $k=1$ to $k=800$ are shown in Table 5.1 .

With an order of accuracy $p=10$, we discretize the boundary with roughly 50 points per wavelength to achieve an accuracy of $10^{-9}$. Table 5.1 shows that the required time scales approximately as $\mathcal{O}\left(k N_{\text {tot }}\right)$. Note that accuracy is lost more or less linearly with increasing wavenumber. The results support our observation above that the fast direct solver is particularly efficient at computing the RCS for multiple angles of incidence, since the cost for each new right-hand side is very modest. In Figure 5.1. we plot the scattered field for a normally incident plane wave at $k=160$, as well as the backscatter RCS for angles ranging from 0 to $\pi$, sampled at 360 equispaced steps. The time taken for the factorization is less than a minute and the total time taken to solve for all incident angles takes around 30 seconds.

Table 5.1: Results for the pot shaped cavity over a range of wavenumbers

\begin{tabular}{|c|c|c|c|c|c|c|}
\hline Wavenumber $k$ & $N_{\text {corner }}$ & $N_{\text {mid }}$ & $N_{\text {tot }}$ & $T_{\text {factor }}$ & $T_{\text {solve }}$ & $E_{\text {error }}$ \\
\hline 1 & 10 & 2 & 1320 & 0.5 & 0.01 & $7.6 \cdot 10^{-10}$ \\
10 & 10 & 10 & 2200 & 0.65 & 0.01 & $5 \cdot 10^{-8}$ \\
20 & 10 & 20 & 3300 & 1.52 & 0.01 & $1.2 \cdot 10^{-9}$ \\
40 & 10 & 40 & 5500 & 3.14 & 0.02 & $8.5 \cdot 10^{-9}$ \\
80 & 10 & 80 & 9900 & 9.5 & 0.05 & $4.1 \cdot 10^{-9}$ \\
160 & 10 & 160 & 18700 & 42.4 & 0.19 & $1.2 \cdot 10^{-9}$ \\
320 & 10 & 320 & 36300 & 192.8 & 0.57 & $2.1 \cdot 10^{-8}$ \\
640 & 10 & 400 & 45100 & 581.4 & 1.43 & $5.5 \cdot 10^{-5}$ \\
800 & 10 & 400 & 45100 & 785.1 & 1.57 & $4.6 \cdot 10^{-6}$ \\
\hline
\end{tabular}




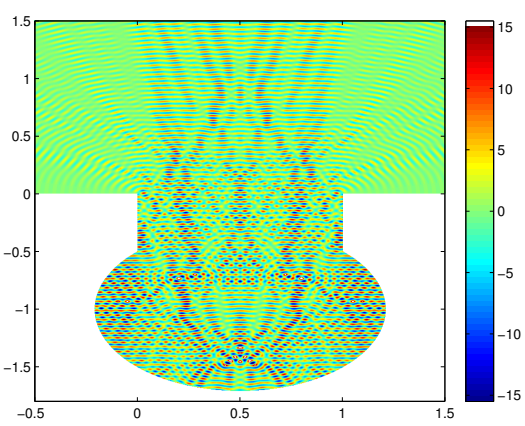

(a)

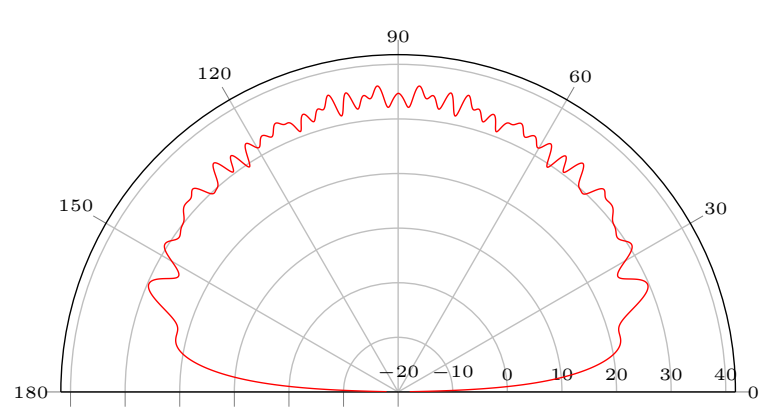

(b)

Fig. 5.1: Example 1. (a) Real part of the scattered field for a pot shaped cavity with a normally incident plane wave at wavenumber $k=160$. (b) The backscatter $R C S$ in $d B$ for the pot shaped cavity at $k=160$

5.2. Example 2: Engine shaped cavity. We next investigate scattering by an engine-shaped cavity. The cavity is constructed by connecting the points $[0,0],[0,-2],[0.45,-2.0],[0.45,-1.6],[0.1,-1.6]$, $[0.1,-1.0],[0.45,-1.0],[0.45,-0.4],[0.5,0.2],[0.55,-0.4],[0.55,-1.0],[0.9,-1.0],[0.9,-1.6],[0.55,-1.6]$, $[0.55,-2.0],[1,-2]$ and $[1,0]$, as shown in Figure 5.2 . Note that the tip of the engine is above the ground plane.

Computational results are shown in Table 5.2. Compared with Example 1, the required solution time is greater for the same wavenumber and approximately the same number of points. This is due to the fact that some segments of the boundary are physically close to each other, which increases the rank of some off-diagonal blocks in the HODLR matrix. The scattered field for a plane wave at angle of incidence $45^{\circ}$ with $k=160$ is plotted in Figure 5.2. The RCS is also shown in Figure 5.2. An animation, Movie 1, of the scattered field as the angle of incidence varies is provided in the supplementary material. Opening the animation using Adobe Reader XI, Version 11.0.06 is recommended.

Table 5.2: Results for engine-shaped cavity over a range of wavenumbers

\begin{tabular}{|c|c|c|c|c|c|c|}
\hline Wavenumber $k$ & $N_{\text {corner }}$ & $N_{\text {mid }}$ & $N_{\text {tot }}$ & $T_{\text {factor }}$ & $T_{\text {solve }}$ & $E_{\text {error }}$ \\
\hline 1 & 10 & 2 & 3360 & 3.1 & 0.01 & $8.8 \cdot 10^{-10}$ \\
10 & 10 & 10 & 5600 & 5.46 & 0.03 & $7.7 \cdot 10^{-12}$ \\
20 & 10 & 20 & 8400 & 8.73 & 0.04 & $5.0 \cdot 10^{-11}$ \\
40 & 10 & 40 & 14000 & 17.83 & 0.1 & $5.9 \cdot 10^{-9}$ \\
80 & 10 & 80 & 25200 & 44.16 & 0.26 & $7.8 \cdot 10^{-8}$ \\
160 & 10 & 160 & 47600 & 155.2 & 0.58 & $4.2 \cdot 10^{-8}$ \\
320 & 10 & 200 & 58800 & 466.3 & 1.27 & $3.3 \cdot 10^{-8}$ \\
640 & 10 & 200 & 58800 & 980.4 & 2.50 & $3.5 \cdot 10^{-8}$ \\
800 & 10 & 200 & 58800 & 1100.1 & 3.01 & $9.6 \cdot 10^{-8}$ \\
\hline
\end{tabular}




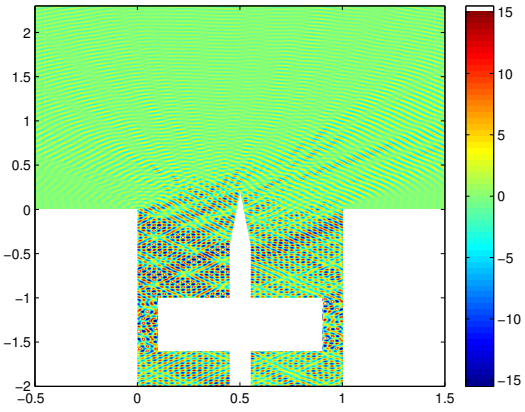

(a)

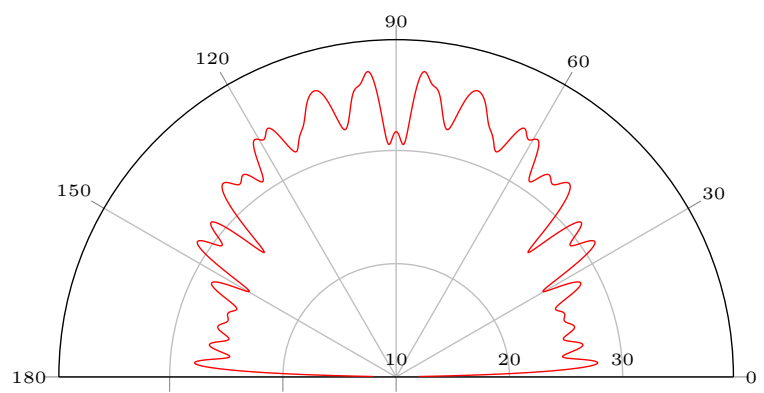

(b)

Fig. 5.2: Example 2: (a) Real part of the scattered field for an engine-shaped cavity in response to an incident plane wave at angle $45^{\circ}$ with wavenumber $k=160$, (b) The backscatter RCS in $d B$ for the engine shaped cavity at $k=160$.

5.3. Example 3: Rough bottom cavity. In our last example, we consider a cavity with a roughshaped boundary, which is extremely difficult to analyze by standard finite difference or finite element methods, especially at large wavenumber. The boundary of the cavity is parametrization by:

$$
\left\{\begin{array}{l}
x(\theta)=r(\theta) \cos (\theta)+0.5 \\
y(\theta)=1.25 r(\theta) \sin (\theta)
\end{array}\right.
$$

where

$$
r(\theta)=1+0.1 \sin (2 \theta)+0.1 \sin (11 \theta)+0.08 \sin (19 \theta) \sin (29 \theta)+0.05 \sin (47 \theta)
$$

and $\theta \in[\pi, 2 \pi]$. Such rough structures may appear in modeling manufacturing defects or during intermediate steps of an optimization procedure aimed at reconstructing an unknown cavity by solving the inverse scattering problem. The boundary is discretized using three segments. The results in Table 5.3 again show that the work is of the order $\mathcal{O}\left(k N_{\text {tot }}\right)$. Figure 5.3 shows the scattered field for $k=160$ with a plane wave at normal incidence, as well as the RCS plot. Note that the rough bottom exhibits a much larger RCS between $\pi / 4$ and $3 \pi / 4$ than in the previous examples.

Table 5.3: Results for rough-bottom cavity over a range of wavenumbers

\begin{tabular}{|c|c|c|c|c|c|c|}
\hline Wavenumber $k$ & $N_{\text {corner }}$ & $N_{\text {mid }}$ & $N_{\text {tot }}$ & $T_{\text {factor }}$ & $T_{\text {solve }}$ & $E_{\text {error }}$ \\
\hline 1 & 10 & 20 & 3300 & 2.3 & 0.01 & $1.3 \cdot 10^{-8}$ \\
10 & 10 & 20 & 3300 & 2.1 & 0.02 & $6.6 \cdot 10^{-9}$ \\
20 & 10 & 40 & 5500 & 3.2 & 0.02 & $5.3 \cdot 10^{-8}$ \\
40 & 10 & 80 & 9900 & 6.5 & 0.05 & $4.8 \cdot 10^{-8}$ \\
80 & 10 & 160 & 18700 & 20.8 & 0.12 & $1.2 \cdot 10^{-8}$ \\
160 & 10 & 320 & 36300 & 78.7 & 0.35 & $5.7 \cdot 10^{-8}$ \\
320 & 10 & 400 & 45100 & 247.3 & 0.73 & $2.9 \cdot 10^{-8}$ \\
640 & 10 & 400 & 45100 & 772.4 & 1.38 & $4.2 \cdot 10^{-7}$ \\
\hline
\end{tabular}




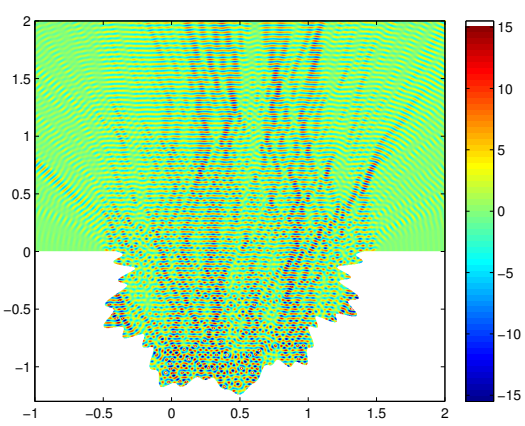

(a)

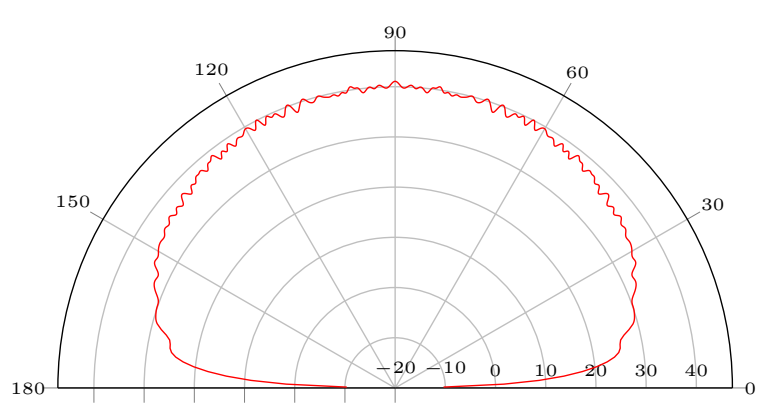

(b)

Fig. 5.3: Example 3. (a) Real part of the scattered field for a rough bottom cavity with a normally incident plane wave at wavenumber $k=160$. (b) The backscatter $R C S$ in $d B$ for the rough bottom cavity at $k=160$.

6. Conclusion. We have presented a new integral formulation for high frequency electromagnetic scattering from a large cavity that leads to a second kind integral equation and which is compatible with a fast and accurate direct solver. The main novelty is the use of a global density, which is non-physical in the sense that the field in certain domains is determined by layer potential densities that are not necessarily on the boundary of the domain itself. We have proven well-posedness of the formulation, which implies that the method does not suffer from any spurious resonances. The equation is discretized by high order quadrature and numerical experiments show that the direct solver is very effective even for large frequencies and arbitrarily-shaped cavities. Future work includes extending the formulation to an impedance boundary, to the full Maxwell equations in three dimensions, and to problems of optimal design.

7. Acknowledgements. This work was supported by the Applied Mathematical Sciences Program of the U.S. Department of Energy under Contract DEFGO288ER25053 and by the Office of the Assistant Secretary of Defense for Research and Engineering and AFOSR under NSSEFF Program Award FA9550-10$1-0180$.

\section{REFERENCES}

[1] B. K. Alpert. Hybrid Gauss-trapezoidal quadrature rules. SIAM J. Sci. Comput., 20:1551-1584, 1999.

[2] S. Ambikasaran. Fast Algorithms for Dense Numerical Linear Algebra and Applications. PhD thesis, Stanford University, 2013.

[3] S. Ambikasaran. A fast direct solver for dense linear systems. https://github.com/sivaramambikasaran/HODLR_Solver, 2013.

[4] S. Ambikasaran and E. Darve. An $\mathcal{O}(N \log N)$ fast direct solver for partial hierarchically semi-separable matrices. Journal of Scientific Computing, pages 1-25, 2013.

[5] A. Aminfar, S. Ambikasaran, and E. Darve. A fast block low-rank dense solver with applications to finite-element matrices. arXiv preprint arXiv:1403.5337 [cs-NA], 2014.

[6] H. Ammari, G. Bao, and A. W. Wood. An integral equation method for the electromagnetic scattering from cavities. Math. Meth. Appl. Sci., 23:1057-1072, 2000.

[7] H. Ammari, G. Bao, and A. W. Wood. Analysis of the electromagnetic scattering from a cavity. Japan J. Indust. Appl. Math., 19(2):301-310, 2002.

[8] H. Ammari, G. Bao, and A. W. Wood. A cavity problem for Maxwell's equation. Meth. Appl. Anal., 9(2):249-260, 2002.

[9] W. Arnoldi. The principle of minimized iterations in the solution of the matrix eigenvalue problem. Quart. Appl. Math, 9(1):17-29, 1951.

[10] J. Asvestas and R. Kleinman. Electromagnetic scattering by indented screens. Antennas and Propagation, IEEE Transactions on, 42(1):22-30, Jan 1994.

[11] G. Bao, J. Gao, J. Lin, and W. Zhang. Mode matching for the electromagnetic scattering from three-dimensional large cavities. IEEE Antennas Wireless Propagat., 60:2004-2010, 2012.

[12] G. Bao and J. Lai. Optimal shape design of a cavity for radar cross section reduction. SIAM J. Control Optim. submitted, 2014.

[13] G. Bao and J. Lai. Radar cross section reduction of a cavity in the ground plane. Commun. Comput. Phys., 15:895-910, 2014. 
[14] G. Bao and W. Sun. A fast algorithm for the electromagnetic scattering from a large cavity. SIAM J. Sci. Comput., 27:553-574, 2005.

[15] G. Bao, K. Yun, and Z. Zhou. Stability of the scattering from a large electromagnetic cavity in two dimensions. SIAM J. Math. Anal., 44(1):383-404, 2012.

[16] G. Bao and W. Zhang. An improved mode-matching method for large cavities. IEEE Antennas Wireless Propagat. Lett., $27: 393-396,2005$.

[17] J. Barnes and P. Hut. A hierarchical $\mathcal{O}(N \log N)$ force-calculation algorithm. Nature, 324(4):446-449, 1986.

[18] M. Bebendorf. Hierarchical LU decomposition-based preconditioners for BEM. Computing, 74(3):225-247, 2005.

[19] S. Börm, L. Grasedyck, and W. Hackbusch. Hierarchical matrices. Lecture notes, 21, 2003.

[20] S. Börm, L. Grasedyck, and W. Hackbusch. Introduction to hierarchical matrices with applications. Engineering Analysis with Boundary Elements, 27(5):405-422, 2003.

[21] J. Bremer. On the Nyström discretization of integral equations on planar curves with corners. Appl. Comput. Harm. Anal., 32:45-64, 2012.

[22] J. Bremer, Z. Gimbutas, and V. Rokhlin. A nonlinear optimization procedure for generalized Gaussian quadratures. SIAM J. Sci. Comput., 32(4):1761-1788, June 2010.

[23] G. A. Chandler. Galerkin's method for boundary integral equations on polygonal domains. J. Austral. Math. Soc. Ser. $B, 26: 1-13,1984$.

[24] S. N. Chandler-wilde and A. T. Peplow. A boundary integral equation formulation for the Helmholtz equation in a locally perturbed half-plane. ZAMM Z. Angew. Math. Mech, pages 79-88, 2005.

[25] S. Chandrasekaran, P. Dewilde, M. Gu, W. Lyons, and T. Pals. A fast solver for HSS representations via sparse matrices. SIAM Journal on Matrix Analysis and Applications, 29(1):67-81, 2006.

[26] S. Chandrasekaran, M. Gu, and T. Pals. A fast ULV decomposition solver for hierarchically semiseparable representations. SIAM Journal on Matrix Analysis and Applications, 28(3):603-622, 2006.

[27] H. Cheng, L. Greengard, and V. Rokhlin. A fast adaptive multipole algorithm in three dimensions. Journal of Computational Physics, 155(2):468-498, 1999.

[28] R. Coifman, V. Rokhlin, and S. Wandzura. The fast multipole method for the wave equation: A pedestrian prescription. Antennas and Propagation Magazine, IEEE, 35(3):7-12, 1993.

[29] D. Colton and R. Kress. Integral Equation Method in Scattering Theory. Wiley-Interscience, New York, 1983.

[30] D. Colton and R. Kress. Inverse Acoustic and Electromagnetic Scattering Theory, Applied Mathematical Sciences 93. Springer-Verlag, Berlin, 1998.

[31] J. Cooley and J. Tukey. An algorithm for the machine calculation of complex Fourier series. Math. Comput, 19(90):297301, 1965.

[32] R. Freund. A transpose-free quasi-minimal residual algorithm for non-Hermitian linear systems. SIAM Journal on Scientific Computing, 14:470, 1993.

[33] R. Freund and N. Nachtigal. QMR: a quasi-minimal residual method for non-Hermitian linear systems. Numerische Mathematik, 60(1):315-339, 1991.

[34] K. L. Greengard, L. Ho and J.-Y. Lee. A fast direct solver for scattering from periodic structures with multiple material interfaces in two dimensions. J. Comput. Phys., 258:738-751, 2014.

[35] L. Greengard, D. Gueyffier, P.-G. Martinsson, and V. Rokhlin. Fast direct solvers for integral equations in complex three-dimensional domains. Acta Numerica, 18(1):243-275, 2009.

[36] L. Greengard and J.-Y. Lee. Stable and accurate integral equation methods for scattering problems with multiple material interfaces in two dimensions. J. Comput. Phys., 231:2389-2395, 2012.

[37] L. Greengard and V. Rokhlin. A fast algorithm for particle simulations. Journal of Computational Physics, 73(2):325-348, 1987.

[38] L. Greengard and V. Rokhlin. A new version of the fast multipole method for the Laplace equation in three dimensions. Acta Numerica, 6(1):229-269, 1997.

[39] W. Hackbusch, L. Grasedyck, and S. Börm. An introduction to hierarchical matrices. Max-Planck-Inst. für Mathematik in den Naturwiss., 2001.

[40] W. Hackbusch and Z. Nowak. On the fast matrix multiplication in the boundary element method by panel clustering. Numerische Mathematik, 54(4):463-491, 1989.

[41] J. Helsing and R. Ojala. Corner singularities for elliptic problems: Integral equations, graded meshes, quadrature, and compressed inverse preconditioning. Journal of Computational Physics, 227(20):8820 - 8840, 2008.

[42] M. Hestenes and E. Stiefel. Methods of conjugate gradients for solving linear systems. Journal of Research of the National Bureau of Standards, 49(6):409-436, 1952.

[43] K. L. Ho and L. Greengard. A fast direct solver for structured linear systems by recursive skeletonization. SIAM Journal on Scientific Computing, 34(5):2507-2532, 2012.

[44] J. Jin. A finite element-boundary integral formulation for scattering by three-dimensional cavity-backed apertures. IEEE Trans. Antennas Propagat., 39:97-104, 1991.

[45] J. Jin. The Finite Element Method in Electromagnetics, 2nd edition. Wiley, New York, 2002.

[46] A. Klöckner, A. Barnett, L. Greengard, and M. O'Neil. Quadrature by expansion: a new method for the evaluation of layer potentials. Journal of Computational Physics, 252:332-349, 2013.

[47] E. Knott, J. Shaeffer, and M. Tuley. Radar Cross Section, Second edition. Scitech Publishing Inc, Releigh, NC, 2004.

[48] W. Y. Kong, J. Bremer, and V. Rokhlin. An adaptive fast direct solver for boundary integral equations in two dimensions. Applied and Computational Harmonic Analysis, 31(3):346-369, 2011.

[49] E. Liberty, F. Woolfe, P.-G. Martinsson, V. Rokhlin, and M. Tygert. Randomized algorithms for the low-rank approximation of matrices. Proceedings of the National Academy of Sciences, 104(51):20167, 2007.

[50] J. Liu and J. Jin. A special higher order finite-element method for scattering by deep cavities. IEEE Trans. Antennas Propagat., 48:694-703, 2000. 
[51] S. Mallat. A theory for multiresolution signal decomposition: The wavelet representation. Pattern Analysis and Machine Intelligence, IEEE Transactions on, 11(7):674-693, 1989.

[52] P.-G. Martinsson. A fast direct solver for a class of elliptic partial differential equations. Journal of Scientific Computing, 38(3):316-330, 2009.

[53] P.-G. Martinsson and V. Rokhlin. A fast direct solver for boundary integral equations in two dimensions. Journal of Computational Physics, 205(1):1-23, 2005.

[54] C. C. Paige and M. A. Saunders. Solution of sparse indefinite systems of linear equations. SIAM Journal on Numerical Analysis, 12(4):617-629, 1975.

[55] S. Rjasanow. Adaptive cross approximation of dense matrices. IABEM 2002, International Association for Boundary Element Methods, 2002.

[56] Y. Saad and M. Schultz. GMRES: A generalized minimal residual algorithm for solving nonsymmetric linear systems. SIAM Journal on Scientific and Statistical Computing, 7(3):856-869, 1986.

[57] H. A. Van der Vorst. BiCGSTAB: A fast and smoothly converging variant of BICG for the solution of nonsymmetric linear systems. SIAM Journal on scientific and Statistical Computing, 13(2):631-644, 1992.

[58] A. Willers and P. Werner. The Helmholtz equation in disturbed half-spaces. Mathematical Methods in the Applied Sciences, 9(1):312-323, 1987.

[59] K. Zhao, M. N. Vouvakis, and J.-F. Lee. The adaptive cross approximation algorithm for accelerated method of moments computations of emc problems. Electromagnetic Compatibility, IEEE Transactions on, 47(4):763-773, 2005. 